\title{
Geminoid: Teleoperated Android of an Existing Person
}

\author{
Shuichi Nishio*, Hiroshi Ishiguro* ${ }^{\dagger}$, Norihiro Hagita* \\ * ATR Intelligent Robotics and Communication Laboratories \\ ${ }^{\dagger}$ Department of Adaptive Machine Systems, Osaka University \\ Japan
}

\section{Introduction}

Why are people attracted to humanoid robots and androids? The answer is simple: because human beings are attuned to understand or interpret human expressions and behaviors, especially those that exist in their surroundings. As they grow, infants, who are supposedly born with the ability to discriminate various types of stimuli, gradually adapt and fine-tune their interpretations of detailed social clues from other voices, languages, facial expressions, or behaviors (Pascalis et al., 2002). Perhaps due to this functionality of nature and nurture, people have a strong tendency to anthropomorphize nearly everything they encounter. This is also true for computers or robots. In other words, when we see PCs or robots, some automatic process starts running inside us that tries to interpret them as human. The media equation theory (Reeves \& Nass, 1996) first explicitly articulated this tendency within us. Since then, researchers have been pursuing the key element to make people feel more comfortable with computers or creating an easier and more intuitive interface to various $\varepsilon$ information devices. This pursuit has also begun spreading in the field of robotics. Recently, 0 researcher's interests in robotics are shifting from traditional studies on navigation and $\stackrel{\oplus}{\subseteq}$ manipulation to human-robot interaction. A number of researches have investigated how ธ 응 understand them (Fong et al., 2003; Breazeal, 2004; Kanda et al., 2004). Many insights from $\Phi$ developmental or cognitive psychologies have been implemented and examined to see how they affect the human response or whether they help robots produce smooth and natural communication with humans.

o However, human-robot interaction studies have been neglecting one issue: the "appearance \& versus behavior problem." We empirically know that appearance, one of the most significant $\underset{0}{0}$ elements in communication, is a crucial factor in the evaluation of interaction (See Figure 1). ॠ The interactive robots developed so far had very mechanical outcomes that do appear as " "robots." Researchers tried to make such interactive robots "humanoid" by equipping them \& with heads, eyes, or hands so that their appearance more closely resembled human beings O and to enable them to make such analogous human movements or gestures as staring, c pointing, and so on. Functionality was considered the primary concern in improving Ф communication with humans. In this manner, many studies have compared robots with different behaviors. Thus far, scant attention has been paid to robot appearances. Although 
there are many empirical discussions on such very simple static robots as dolls, the design of a robot's appearance, particularly to increase its human likeness, has always been the role of industrial designers; it has seldom been a field of study. This is a serious problem for developing and evaluating interactive robots. Recent neuroimaging studies show that certain brain activation does not occur when the observed actions are performed by nonhuman agents (Perani et al., 2001; Han et al., 2005). Appearance and behavior are tightly coupled, and concern is high that evaluation results might be affected by appearance.

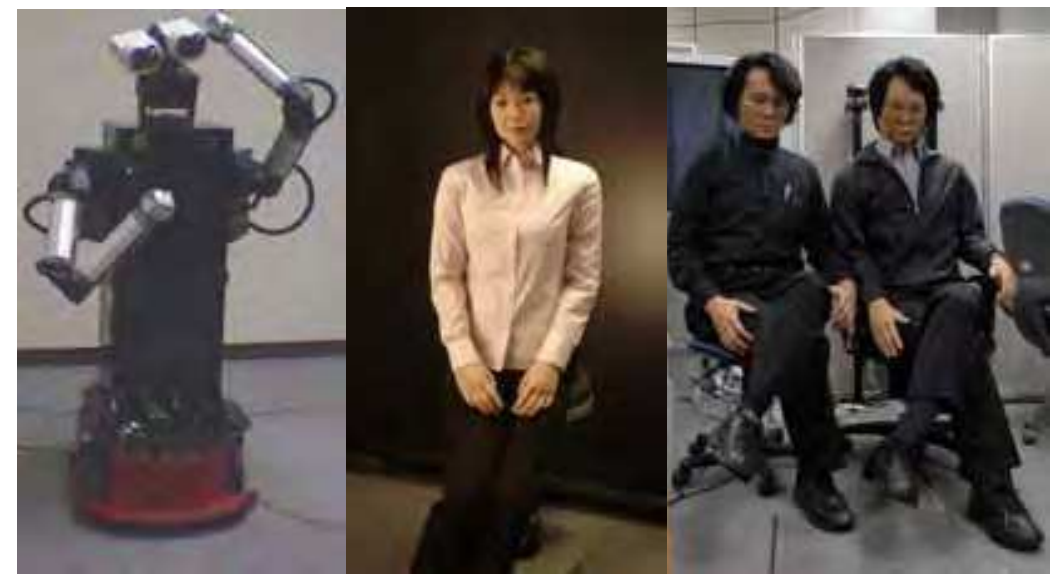

Fig. 1. Three categories of humanlike robots: humanoid robot Robovie II (left: developed by ATR Intelligent Robotics and Communication Laboratories), android Repliee Q2 (middle: developed by Osaka University and Kokoro corporation), geminoid HI-1 and its human source (right: developed by ATR Intelligent Robotics and Communication Laboratories).

In this chapter, we introduce android science, a cross-interdisciplinary research framework that combines two approaches, one in robotics for constructing very humanlike robots and androids, and another in cognitive science that uses androids to explore human nature. Here androids serve as a platform to directly exchange insights from the two domains. To proceed with this new framework, several androids have been developed so far, and many researches have been done. At that time, however, we encountered serious issues that sparked the development of a new category of robot called geminoid. Its concept and the development of the first prototype are described. Preliminary findings to date and future directions with geminoids are also discussed.

\section{Android Science}

Current robotics research uses various findings from the field of cognitive science, especially in the human-robot interaction area, trying to adopt findings from human-human interactions with robots to make robots that people can easily communicate with. At the same time, cognitive science researchers have also begun to utilize robots. As research fields extend to more complex, higher-level human functions such as seeking the neural basis of social skills (Blakemore, 2004), expectations will rise for robots to function as easily controlled apparatuses with communicative ability. However, the contribution from robotics to cognitive science has not been adequate because the appearance and behavior of 
current robots cannot be separately handled. Since traditional robots look quite mechanical and very different from human beings, the effect of their appearance may be too strong to ignore. As a result, researchers cannot clarify whether a specific finding reflects the robot's appearance, its movement, or a combination of both.

We expect to solve this problem using an android whose appearance and behavior closely resembles humans. The same thing is also an issue in robotics research, since it is difficult to clearly distinguish whether the cues pertain solely to robot behaviors. An objective, quantitative means of measuring the effect of appearance is required.

Androids are robots whose behavior and appearance are highly anthropomorphized. Developing androids requires contributions from both robotics and cognitive science. To realize a more humanlike android, knowledge from human sciences is also necessary. At the same time, cognitive science researchers can exploit androids for verifying hypotheses in understanding human nature. This new, bi-directional, crossinterdisciplinary research framework is called android science (Ishiguro, 2005). Under this framework, androids enable us to directly share knowledge between the development of androids in engineering and the understanding of humans in cognitive science (Figure 2).

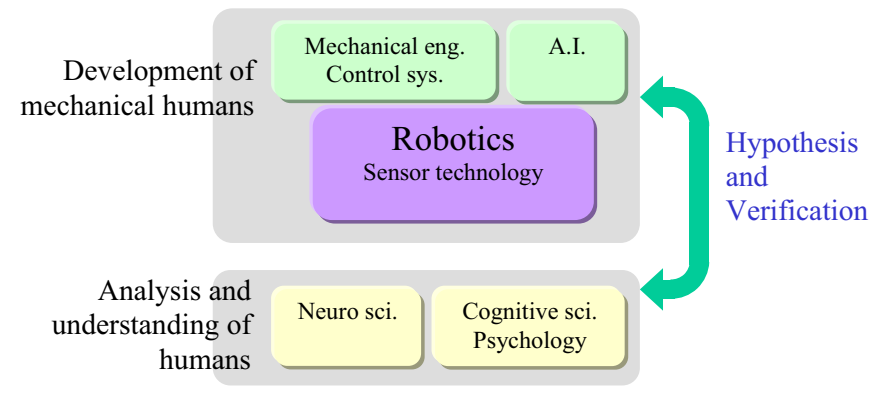

Fig. 2. Bi-directional feedback in Android Science.

The major robotics issue in constructing androids is the development of humanlike appearance, movements, and perception functions. On the other hand, one issue in cognitive science is "conscious and unconscious recognition." The goal of android science is to realize a humanlike robot and to find the essential factors for representing human likeness. How can we define human likeness? Further, how do we perceive human likeness? It is common knowledge that humans have conscious and unconscious recognition. When we observe objects, various modules are activated in our brain. Each of them matches the input sensory data with human models, and then they affect reactions. A typical example is that even if we recognize a robot as an android, we react to it as a human. This issue is fundamental both for engineering and scientific approaches. It will be an evaluation criterion in android development and will provide cues for understanding the human brain's mechanism of recognition.

So far, several androids have been developed. Repliee Q2, the latest android (Ishiguro, 2005), is shown in the middle of Figure 1. Forty-two pneumatic actuators are embedded in the android's upper torso, allowing it to move smoothly and quietly. Tactile sensors, which are also embedded under its skin, are connected to sensors in its environment, such as omnidirectional cameras, microphone arrays, and floor sensors. Using these sensory inputs, 
the autonomous program installed in the android can make smooth, natural interactions with people near it.

Even though these androids enabled us to conduct a variety of cognitive experiments, they are still quite limited. The bottleneck in interaction with human is its lack of ability to perform long-term conversation. Unfortunately, since current AI technology for developing humanlike brains is limited, we cannot expect humanlike conversation with robots. When meeting humanoid robots, people usually expect humanlike conversation with them. However, the technology greatly lags behind this expectation. AI progress takes time, and such AI that can make humanlike conversation is our final goal in robotics. To arrive at this final goal, we need to use currently available technologies and understand deeply what a human is. Our solution for this problem is to integrate android and teleoperation technologies.

\section{Geminoid}
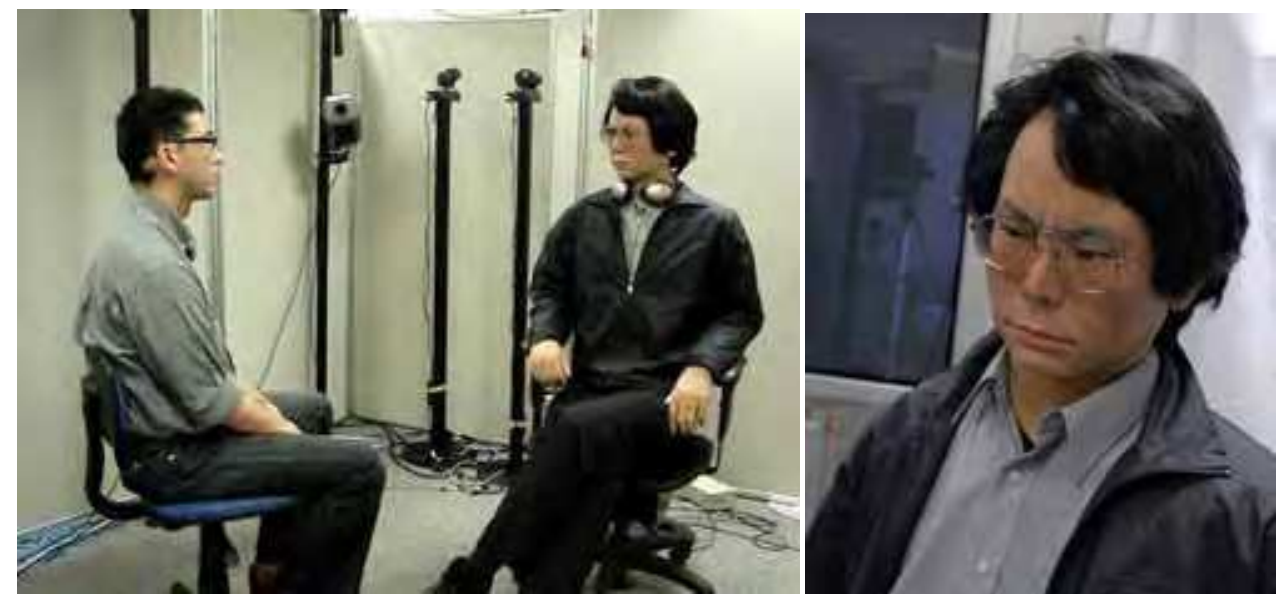

Fig. 3. Geminoid HI-1 (right).

We have developed Geminoid, a new category of robot, to overcome the bottleneck issue. We coined "geminoid" from the Latin "geminus," meaning "twin" or "double," and added "oides," which indicates "similarity" or being a twin. As the name suggests, a geminoid is a robot that will work as a duplicate of an existing person. It appears and behaves as a person and is connected to the person by a computer network. Geminoids extend the applicable field of android science. Androids are designed for studying human nature in general. With geminoids, we can study such personal aspects as presence or personality traits, tracing their origins and implemention into robots. Figure 3 shows the robotic part of HI- 1 , the first geminoid prototype. Geminoids have the following capabilities:

\section{Appearance and behavior highly similar to an existing person}

The appearance of a geminoid is based on an existing person and does not depend on the imagination of designers. Its movements can be made or evaluated simply by referring to the original person. The existence of a real person analogous to the robot enables easy comparison studies. Moreover, if a researcher is used as the original, we can expect that 
individual to offer meaningful insights into the experiments, which are especially important at the very first stage of a new field of study when beginning from established research methodologies.

\section{Teleoperation (remote control)}

Since geminoids are equipped with teleoperation functionality, they are not only driven by an autonomous program. By introducing manual control, the limitations in current AI technologies can be avoided, enabling long-term, intelligent conversational human-robot interaction experiments. This feature also enables various studies on human characteristics by separating "body" and "mind." In geminoids, the operator (mind) can be easily exchanged, while the robot (body) remains the same. Also, the strength of connection, or what kind of information is transmitted between the body and mind, can be easily reconfigured. This is especially important when taking a top-down approach that adds/deletes elements from a person to discover the "critical" elements that comprise human characteristics. Before geminoids, this was impossible.

\subsection{System overview}

The current geminoid prototype, HI-1, consists of roughly three elements: a robot, a central controlling server (geminoid server), and a teleoperation interface (Figure 4).

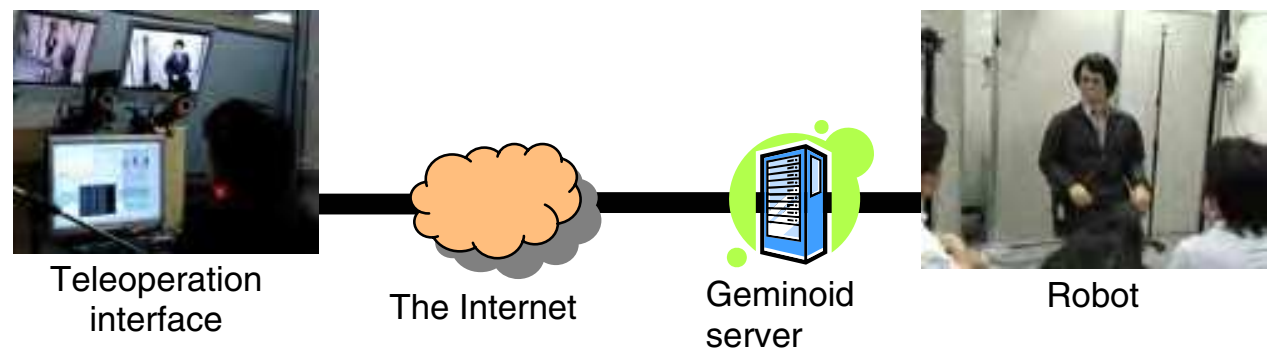

Fig. 4. Overview of geminoid system.

\section{A robot that resembles a living person}

The robotic element has essentially identical structure as previous androids (Ishiguro, 2005). However, efforts concentrated on making a robot that appears - not just to resemble a living person - to be a copy of the original person. Silicone skin was molded by a cast taken from the original person; shape adjustments and skin textures were painted manually based on MRI scans and photographs. Fifty pneumatic actuators drive the robot to generate smooth and quiet movements, which are important attributes when interacting with humans. The allocations of actuators were decided so that the resulting robot can effectively show the necessary movements for human interaction and simultaneously express the original person's personality traits. Among the 50 actuators, 13 are embedded in the face, 15 in the torso, and the remaining 22 move the arms and legs. The softness of the silicone skin and the compliant nature of the pneumatic actuators also provide safety while interacting with humans. Since this prototype was aimed for interaction experiments, it lacks the capability to walk around; it always remains seated. Figure 1 shows the resulting robot (right) alongside the original person, Dr. Ishiguro (author). 


\section{Teleoperation interface}

Figure 5 shows the teleoperation interface prototype. Two monitors show the controlled robot and its surroundings, and microphones and a headphone are used to capture and transmit utterances. The captured sounds are encoded and transmitted to the geminoid server by IP links from the interface to the robot and vice versa. The operator's lip corner positions are measured by an infrared motion capturing system in real time, converted to motion commands, and sent to the geminoid server by the network. This enables the operator to implicitly generate suitable lip movement on the robot while speaking. However, compared to the large number of human facial muscles used for speech, the current robot only has a limited number of actuators on its face. Also, response speed is much slower, partially due to the nature of the pneumatic actuators. Thus, simple transmission and playback of the operator's lip movement would not result in sufficient, natural robot motion. To overcome this issue, measured lip movements are currently transformed into control commands using heuristics obtained through observation of the original person's actual lip movement.
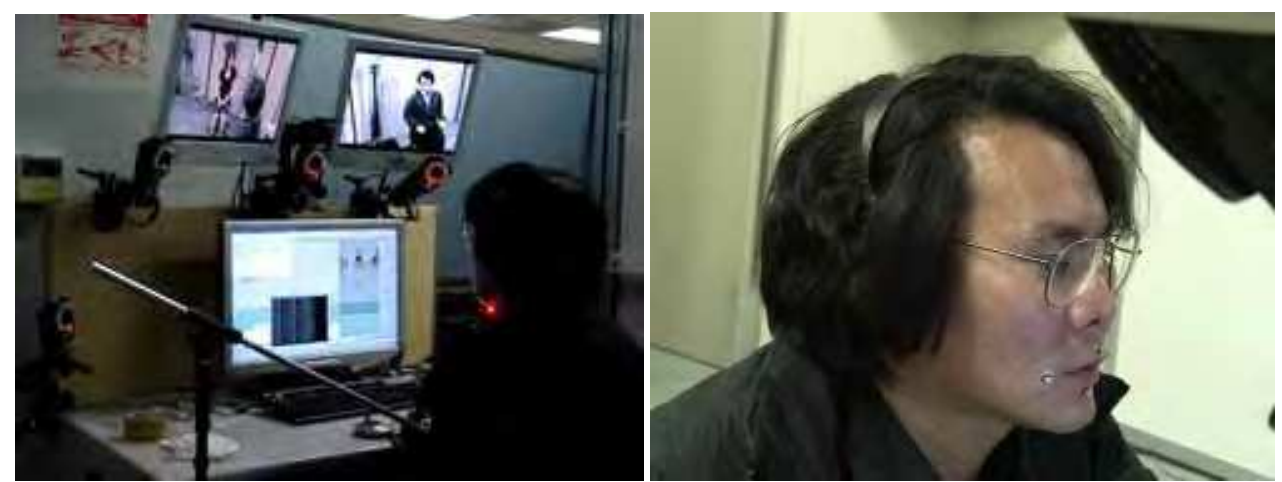

Fig. 5. Teleoperation interface.

The operator can also explicitly send commands for controlling robot behaviors using a simple GUI interface. Several selected movements, such as nodding, opposing, or staring in a certain direction can be specified by a single mouse click. This relatively simple interface was prepared because the robot has 50 degrees of freedom, which makes it one of the world's most complex robots, and is basically impossible to manipulate manually in real time. A simple, intuitive interface is necessary so that the operator can concentrate on interaction and not on robot manipulation. Despite its simplicity, by cooperating with the geminoid server, this interface enables the operator to generate natural humanlike motions in the robot.

\section{Geminoid server}

The geminoid server receives robot control commands and sound data from the remote controlling interface, adjusts and merges inputs, and sends and receives primitive controlling commands between the robot hardware. Figure 6 shows the data flow in the geminoid system. The geminoid server also maintains the state of human-robot interaction and generates autonomous or unconscious movements for the robot. As described above, as the robot's features become more humanlike, its behavior should also become suitably 
sophisticated to retain a "natural" look (Minato et al., 2006). One thing that can be seen in every human being, and that most robots lack, are the slight body movements caused by an autonomous system, such as breathing or blinking. To increase the robot's naturalness, the geminoid server emulates the human autonomous system and automatically generates these micro-movements, depending on the state of interaction each time. When the robot is "speaking," it shows different micro-movements than when "listening" to others. Such automatic robot motions, generated without operator's explicit orders, are merged and adjusted with conscious operation commands from the teleoperation interface (Figure 6). Alongside, the geminoid server gives the transmitted sounds specific delay, taking into account the transmission delay/jitter and the start-up delay of the pneumatic actuators. This adjustment serves synchronizing lip movements and speech, thus enhancing the naturalness of geminoid movement.

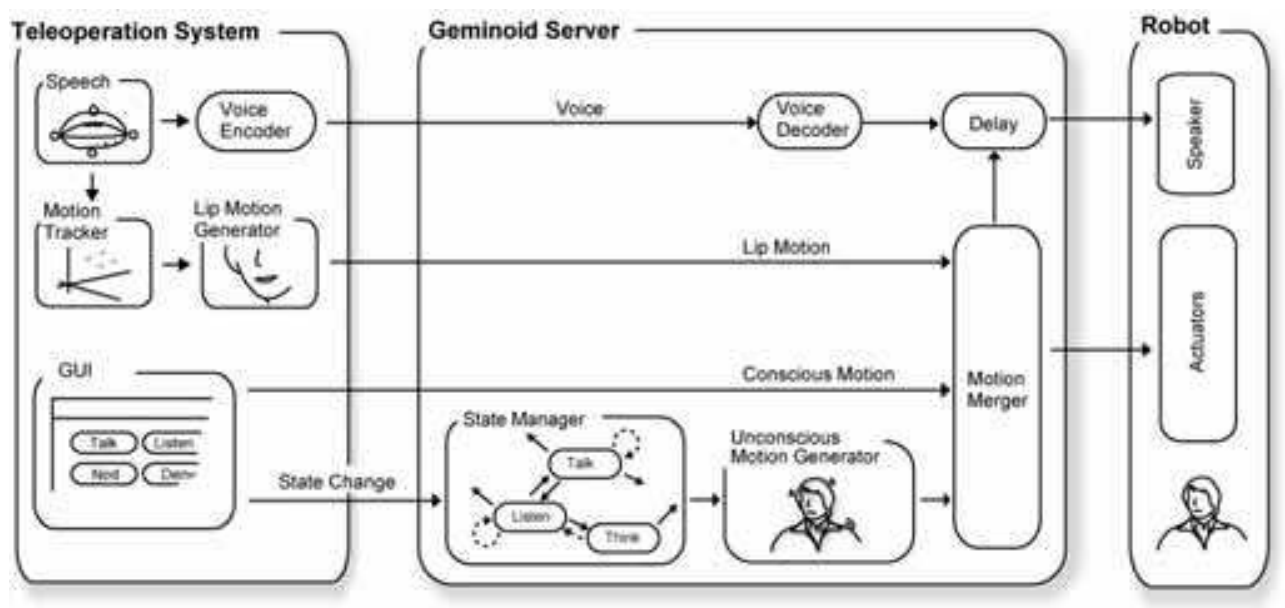

Fig. 6. Data flow in the geminoid system.

\subsection{Experiences with the geminoid prototype}

The first geminoid prototype, HI-1, was completed and press-released in July 2006. Since then, numerous operations have been held, including interactions with lab members and experiment subjects. Also, geminoid was demonstrated to a number of visitors and reporters. During these operations, we encountered several interesting phenomena. Here are some discourses made by the geminoid operator:

- When I (Dr. Ishiguro, the origin of the geminoid prototype) first saw HI-1 sitting still, it was like looking in a mirror. However, when it began moving, it looked like somebody else, and I couldn't recognize it as myself. This was strange, since we copied my movements to HI-1, and others who know me well say the robot accurately shows my characteristics. This means that we are not objectively recognizing our unconscious movements ourselves.

- While operating HI-1 with the operation interface, I find myself unconsciously adapting my movements to the geminoid movements. The current geminoid cannot move as freely as I can. I felt that, not just the geminoid but my own body is restricted to the movements that HI-1 can make. 
- In less than 5 minutes both the visitors and I can quickly adapt to conversation through the geminoid. The visitors recognize and accept the geminoid as me while talking to each other.

- When a visitor pokes HI-1, especially around its face, I get a strong feeling of being poked myself. This is strange, as the system currently provides no tactile feedback. Just by watching the monitors and interacting with visitors, I get this feeling.

We also asked the visitors how they felt when interacting through the geminoid. Most said that when they saw HI-1 for the very first time, they thought that somebody (or Dr. Ishiguro, if familiar with him) was waiting there. After taking a closer look, they soon realized that HI-1 was a robot and began to have some weird and nervous feelings. But shortly after having a conversation through the geminoid, they found themselves concentrating on the interaction, and soon the strange feelings vanished. Most of the visitors were nonresearchers unfamiliar with robots of any kind.

Does this mean that the geminoid has overcome the "uncanny valley"? Before talking through the geminoid, the initial response of the visitors seemingly resembles the reactions seen with previous androids: even though at the very first moment they could not recognize the androids as artificial, they nevertheless soon become nervous while being with the androids. Are intelligence or long-term interaction crucial factors in overcoming the valley and arriving at an area of natural humanness?

We certainly need objective means to measure how people feel about geminoids and other types of robots. In a previous android study, Minato et al. found that gaze fixation revealed criteria about the naturalness of robots (Minato et al., 2006). Recent studies have shown different human responses and reactions to natural or artificial stimuli of the same nature. Perani et al. showed that different brain regions are activated while watching human or computer graphic arms movements (Perani et al., 2001). Kilner et al. showed that body movement entrainment occurs when watching human motions, but not with robot motions (Kilner et al., 2003). By examining these findings with geminoids, we may be able to find some concrete measurements of human likeliness and approach the "appearance versus behavior" issue.

Perhaps HI-1 was recognized as a sort of communication device, similar to a telephone or a TV-phone. Recent studies have suggested a distinction in the brain process that discriminates between people appearing in videos and existing persons appearing live (Kuhl et al., 2003). While attending TV conferences or talking by cellular phones, however, we often experience the feeling that something is missing from a face-to-face meeting. What is missing here? Is there an objective means to measure and capture this element? Can we ever implement this on robots?

\section{Summary and further issues}

In developing the geminoid, our purpose is to study Sonzai-Kan, or human presence, by extending the framework of android science. The scientific aspect must answer questions about how humans recognize human existence/presence. The technological aspect must realize a teleoperated android that works on behalf of the person remotely accessing it. This will be one of the practical networked robots realized by integrating robots with the Internet.

The following are our current challenges: 
Teleoperation technologies for complex humanlike robots

Methods must be studied to teleoperate the geminoid to convey existence/presence, which is much more complex than traditional teleoperation for mobile and industrial robots. We are studying a method to autonomously control an android by transferring motions of the operator measured by a motion capturing system. We are also developing methods to autonomously control eye-gaze and humanlike small and large movements.

Synchronization between speech utterances sent by the teleoperation system and body movements

The most important technology for the teleoperation system is synchronization between speech utterances and lip movements. We are investigating how to produce natural behaviors during speech utterances. This problem is extended to other modalities, such as head and arm movements. Further, we are studying the effects on non-verbal communication by investigating not only synchronization of speech and lip movements but also facial expressions, head, and even whole body movements.

\section{Psychological test for human existence/presence}

We are studying the effect of transmitting Sonzai-Kan from remote places, such as meeting participation instead of the person himself. Moreover, we are interested in studying existence/presence through cognitive and psychological experiments. For example, we are studying whether the android can represent the authority of the person himself by comparing the person and the android.

\section{Application}

Although being developed as research apparatus, the nature of geminoids can allow us to extend the use of robots in the real world. The teleoperated, semi-autonomous facility of geminoids allows them to be used as substitutes for clerks, for example, that can be controlled by human operators only when non-typical responses are required. Since in most cases an autonomous AI response will be sufficient, a few operators will be able to control hundreds of geminoids. Also because their appearance and behavior closely resembles humans, in the next age geminoids should be the ultimate interface device.

\section{Acknowledgement}

This work was supported in part by the Ministry of Internal Affairs and Communications of Japan.

\section{References}

Blakemore, S. J. \& Frith, U. (2004). How does the brain deal with the social world? Neuroreport, 15, 119-128

Breazeal, C. (2004). Social Interactions in HRI: The Robot View, IEEE Transactions on Man, Cybernetics and Systems: Part C, 34, 181-186

Fong, T., Nourbakhsh, I., \& Dautenhahn, K. (2003). A survey of socially interactive robots, Robotics and Autonomous Systems, 42, 143-166 
Han, S., Jiang, Y., Humphreys, G. W., Zhou, T., and \& Cai, P. (2005). Distinct neural substrates for the perception of real and virtual visual worlds, NeuroImage, 24, 928- 935

Ishiguro, H. (2005). Android Science: Toward a New Cross-Disciplinary Framework. Proceedings of Toward Social Mechanisms of Android Science: A CogSci 2005 Workshop, 1-6

Kanda, T., Ishiguro, H., Imai, M., \& Ono, T. (2004). Development and Evaluation of Interactive Humanoid Robots, Proceedings of the IEEE, 1839-1850

Kilner, J. M., Paulignan, Y., \& Blakemore, S. J. (2003). An interference effect of observed biological movement on action, Current Biology, 13, 522-525

Kuhl, P. K., Tsao. F. M., \& Liu, H. M. (2003). Foreign-language experience in infancy: Effects of short-term exposure and social interaction on phonetic learning. Proceedings of the National Academy of Sciences, 100, 9096-9101

Minato, T., Shimada, M., Itakura, S., Lee, K., \& Ishiguro, H. (2006). Evaluating the human likeness of an android by comparing gaze behaviors elicited by the android and a person, Advanced Robotics, 20, 1147-1163

Pascalis, O., Haan, M., and Nelson, C. A. (2002). Is Face Processing Species-Specific During the First Year of Life?, Science, 296, 1321-1323

Perani, D., Fazio, F., Borghese, N. A., Tettamanti, M., Ferrari, S., Decety, J., \& Gilardi, M.C. (2001). Different brain correlates for watching real and virtual hand actions, NeuroImage, 14, 749-758

Reeves, B. \& Nass, C. (1996). The Media Equation, CSLI/Cambridge University Press 


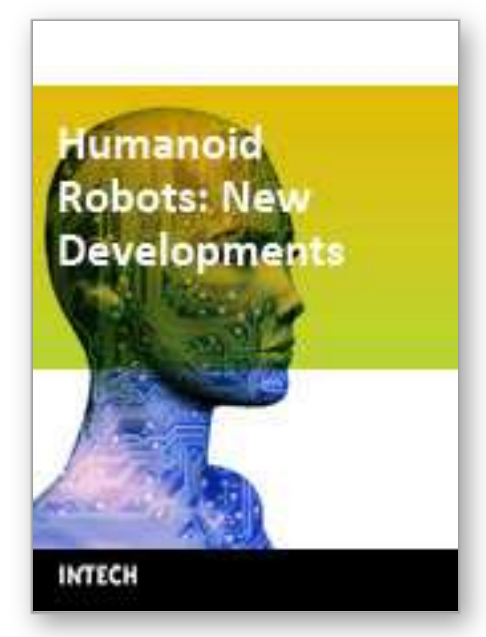

\author{
Humanoid Robots: New Developments \\ Edited by Armando Carlos de Pina Filho
}

ISBN 978-3-902613-00-4

Hard cover, 582 pages

Publisher I-Tech Education and Publishing

Published online 01, June, 2007

Published in print edition June, 2007

For many years, the human being has been trying, in all ways, to recreate the complex mechanisms that form the human body. Such task is extremely complicated and the results are not totally satisfactory. However, with increasing technological advances based on theoretical and experimental researches, man gets, in a way, to copy or to imitate some systems of the human body. These researches not only intended to create humanoid robots, great part of them constituting autonomous systems, but also, in some way, to offer a higher knowledge of the systems that form the human body, objectifying possible applications in the technology of rehabilitation of human beings, gathering in a whole studies related not only to Robotics, but also to Biomechanics, Biomimmetics, Cybernetics, among other areas. This book presents a series of researches inspired by this ideal, carried through by various researchers worldwide, looking for to analyze and to discuss diverse subjects related to humanoid robots. The presented contributions explore aspects about robotic hands, learning, language, vision and locomotion.

\title{
How to reference
}

In order to correctly reference this scholarly work, feel free to copy and paste the following:

Shuichi Nishio, Hiroshi Ishiguro and Norihiro Hagita (2007). Geminoid: Teleoperated Android of an Existing Person, Humanoid Robots: New Developments, Armando Carlos de Pina Filho (Ed.), ISBN: 978-3-902613-004, InTech, Available from:

http://www.intechopen.com/books/humanoid_robots_new_developments/geminoid_teleoperated_android_of _an_existing_person

\section{INTECH}

open science | open minds

\author{
InTech Europe \\ University Campus STeP Ri \\ Slavka Krautzeka 83/A \\ 51000 Rijeka, Croatia \\ Phone: +385 (51) 770447 \\ Fax: +385 (51) 686166 \\ www.intechopen.com
}

\author{
InTech China \\ Unit 405, Office Block, Hotel Equatorial Shanghai \\ No.65, Yan An Road (West), Shanghai, 200040, China \\ 中国上海市延安西路65号上海国际贵都大饭店办公楼405单元 \\ Phone: +86-21-62489820 \\ Fax: +86-21-62489821
}


(C) 2007 The Author(s). Licensee IntechOpen. This chapter is distributed under the terms of the Creative Commons Attribution-NonCommercial-ShareAlike-3.0 License, which permits use, distribution and reproduction for non-commercial purposes, provided the original is properly cited and derivative works building on this content are distributed under the same license. 